\title{
Mitral valve leaflet length as an important factor to differentiate hypertrophic cardiomyopathy from other causes of left ventricular hypertrophy
}

\author{
Yong Luo ${ }^{1 *}$, Dan Yang ${ }^{1}$, Hong Liu', Ke Wan ${ }^{1}$, Jiayu Sun², Tianjing Zhang ${ }^{3}$, Yucheng Chen ${ }^{1}$ \\ From 19th Annual SCMR Scientific Sessions \\ Los Angeles, CA, USA. 27-30 January 2016
}

\section{Background}

Left ventricular hypertrophy (LVH) with Left ventricular maximal thickness (LVMT) $\geq 15 \mathrm{~mm}$ as a current criteria to differentiate hypertrophic cardiomyopathy (HCM) from other causes of LVH lacks specificity. The recent findings of intrinsic mitral valve leaflet elongation in HCM may potentially distinguish HCM from other causes of LVH. We aim to studying the performance of mitral valve leaflet length in differential diagnosis of HCM from other diseases with $\mathrm{LVH}$.

\section{Methods}

Contrast-enhanced cardiac magnetic resonance was performed following a standard protocol in 291 subjects (grouped by $112 \mathrm{HCM}$ patients; 89 patients with LVH secondary to hypertension, cardiac amyloidosis or aortic stenosis; and 90 controls). Differences in anterior and posterior mitral leaflet length (AML and PML) (mm) among groups were analyzed, as were the diagnostic performance of AML or PML in distinguishing HCM from non HCM LVH.

\section{Results}

AML and PML in HCM group were longest among the three groups (AML: HCM $26.49 \pm 4.28$ vs. non HCM LVH $21.98 \pm 3.57$ VS. normal $21.10 \pm 3.12$, p < 0.001; PML: HCM $13.57 \pm 3.12$ vs. non HCM LVH $11.47 \pm 2.35$ vs. normal $11.72 \pm 2.89, \mathrm{p}<0.001)$. Furthermore, AML and PML were elongating only in HCM regardless of division by $\operatorname{LVMT}(<15 \mathrm{~mm}$ and $\geq 15 \mathrm{~mm})$ or hypertrophic pattern (symmetric and asymmetric) $(\mathrm{p}<0.05)$. Multiple logistic regression analysis showed AML, PML and LVMT were predictors to differentiate HCM from non HCM $\mathrm{LVH}$ with $\mathrm{LVMT} \geq 15 \mathrm{~mm}(\mathrm{p}<0.05)$. When performed ROC, LVMT $>21 \mathrm{~mm}$ was proved an optimal cutoff value to differentiate HCM from non HCM LVH with LVMT $\geq 15$ mm (AUC 0.779 [95\%CI 0.708-0.839]; sensitivity $61.6 \%$, specificity $85.71 \%)$. In patients with LVMT between $15 \mathrm{~mm}$ and $21 \mathrm{~mm}$, AML $>24 \mathrm{~mm}$ and $\mathrm{PML}>13$ $\mathrm{mm}$ showed similarly favorable differential diagnosis value between HCM and non HCM LVH by ROC curves (AML: AUC 0.761 [95\%CI 0.660-0.844], sensitivity 67.4\%, specificity 81.2\%; PML: AUC 0.737 [95\%CI 0.634-0.823]; sensitivity $51.2 \%$, specificity $89.6 \%$ ).

\section{Conclusions}

Elongation of mitral leaflet length in $\mathrm{HCM}$ is a specific and intrinsic characteristic and it has a favorable differential value to discriminate HCM from non HCM diseases.

\section{Authors' details}

${ }^{1}$ Cardiology Department, West China Hospital, Chengdu, China. ${ }^{2}$ Radiology Department, Westchina Hospital, Chengdu, China. ${ }^{3}$ Siemens MR Northeastern Collaboration, Beijing, China.

Published: 27 January 2016

doi:10.1186/1532-429X-18-S1-P272

Cite this article as: Luo et al:: Mitral valve leaflet length as an important factor to differentiate hypertrophic cardiomyopathy from other causes of left ventricular hypertrophy. Journal of Cardiovascular Magnetic Resonance 2016 18(Suppl 1):P272.

${ }^{1}$ Cardiology Department, West China Hospital, Chengdu, China

Full list of author information is available at the end of the article

(C) 2016 Luo et al. This is an Open Access article distributed under the terms of the Creative Commons Attribution License (http:// creativecommons.org/licenses/by/4.0), which permits unrestricted use, distribution, and reproduction in any medium, provided the original work is properly cited. The Creative Commons Public Domain Dedication waiver (http://creativecommons.org/publicdomain/ zero/1.0/) applies to the data made available in this article, unless otherwise stated. 